\title{
What about Ionic Liquids as a "hot" Certified Reference Material Candidate to check Your EDS below 1 keV?
}

\author{
Markus Holzweber ${ }^{1}$, Vasile-Dan Hodoroaba ${ }^{1}$ and Wolfgang E. S. Unger ${ }^{1}$ \\ 1. BAM Federal Institute for Materials Research and Testing, Division 6.8 Surface Analysis and \\ Interfacial Chemistry, Unter den Eichen 44, 12200 Berlin, Germany.
}

Energy-dispersive X-ray spectrometry (EDX) is one of the most applied methods used for the analysis of the chemical composition of solids and thin films. Recent progress in EDS (Energy Dispersive X-ray Spectrometer) technology has increased the general performance also in the energy range $<1 \mathrm{keV}$ addressing low $\mathrm{Z}$ elements. Suitable test materials to be employed especially to check the low-energy EDS performance - also in line with ISO 15632 [1] - are rather limited and mainly based on C K and F $\mathrm{K}$ lines [2]. In order to obtain valid results in laboratories accredited in compliance with ISO/IEC 17025 [3] it is necessary to periodically check the instrument performance.

Room temperature ionic liquids (RTIL) are composed of ions only and are defined as molten salts with a melting point below $100^{\circ} \mathrm{C}$. [4] They have numerous interesting physical properties such as high thermal stability and electrical conductivity. Besides their low melting points they have also a very low vapor pressure, which enables their analysis even in ultra -high vacuum devices.

In this contribution we explore the applicability of RTIL for the routine test check of the energy scale and the energy resolution. RTILs fulfil in principle the general requirements to qualify them as a reference material: RTILs can easily be prepared reproducibly to exhibit a smooth and flat surface, see Figure 1, show excellent lateral and in-depth homogeneity, are electrically conductive in the liquid state and are long-term stable at specific storage conditions (e.g. low temperature, moisture free). Additionally, the surface is regenerating itself. By varying the alkyl side chain length different relative elemental concentration can be obtained easily.

As a proof-of-principle we investigated RTILs of the imidazolium bis(trifluoromethyl)sulfonylimide class with variable carbon content (i.e. different side chain length). We choose this class of RTILs since they are readily available in high purity and contain light elements $(\mathrm{C}, \mathrm{N}, \mathrm{O}, \mathrm{F})$ which offer several $\mathrm{X}$ ray characteristic $\mathrm{K}$ line peaks below $1 \mathrm{keV}$ besides the $\mathrm{S} \mathrm{K}$ line above $1 \mathrm{keV}$. The capability of an EDS system to detect S L series at $148.7 \mathrm{eV}$ can also be evaluated. Examples of EDX spectra taken from an RTIL reference material candidate are shown in Figure 1. The differences in the energy resolution of the two EDS systems used here in the low-energy range are expressed by different half widths (FWHM) of the lines. This has an effect on the separation of the N K lines.

Furthermore, direct comparisons of EDX spectra taken under identical conditions by EDS with different performances in the low energy range can quickly distinguish differences not only in the energy resolution but also in the spectrometer efficiency. At a later stage, this new candidate can be used as a sensitive test material to validate the quantification of $\mathrm{X}$-ray lines below $1 \mathrm{keV}$. 


\section{References:}

[1] ISO 15632 (2012) Microbeam analysis - Selected instrumental performance parameters for the specification and checking of energy-dispersive X-ray spectrometersEDX for use in electron probe microanalysisEPMA. ISO, Geneva.

[2] V Rackwitz, M Krumrey, C Laubis et al., Anal Bioanal Chem, in press, DOI 10.1007/s00216-014$8242-5$.

[3] ISO/IEC 17025 (2005) General requirements for the competence of testing and calibration laboratories. ISO, Geneva.

[4] P Wasserscheid and T Welton, Ionic Liquids in Synthesis (2002) (Wiley-VCH, Weinheim).

[5] This work is funded by the European Union through the European Metrology Research Program (EMRP). The EMRP is jointly funded by the EMRP participating countries within EURAMET and the European Union. M.H. is grateful for financial support by the Austrian Science Found (FWF) through the Erwin-Schrödinger fellowship program (project number J 3471-N28).

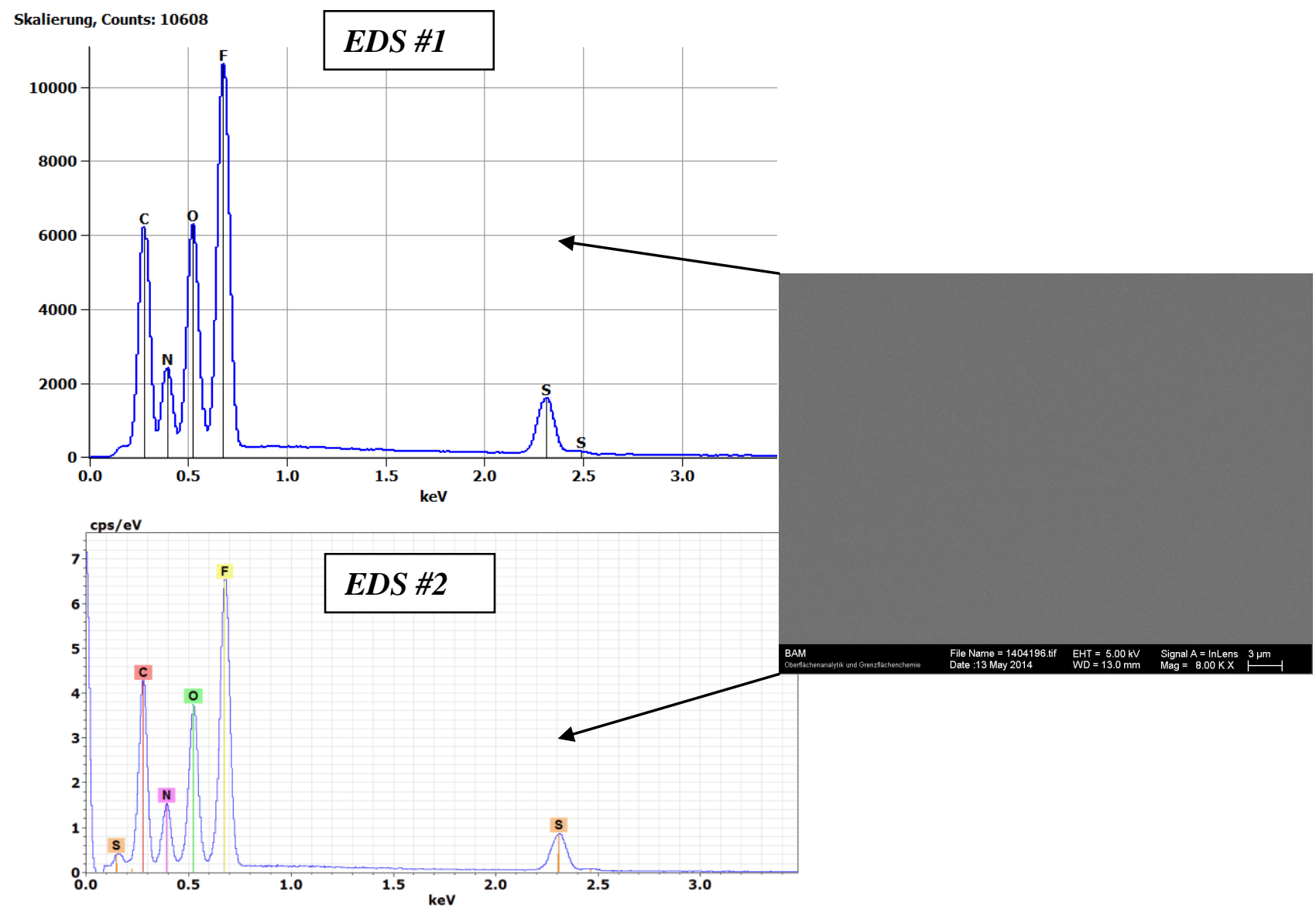

Figure 1: Example of a RTIL test material candidate measured with two different SDD EDS spectrometers under similar conditions. Note the superior energy resolution of the second EDS system (see the better separation of $\mathrm{N} \mathrm{K}$ line). The excellent surface homogeneity can be taken from the SEM micrograph. 